TI 2003-052/4

Tinbergen Institute Discussion Paper

\title{
Measuring Synchronisation and Convergence of Business Cycles
}

Siem Jan Koopman',2

Joao Valle e Azevedo'

I Faculty of Economics and Business Administration, Vrije Universiteit Amsterdam,

2 Tinbergen Institute. 


\section{Tinbergen Institute}

The Tinbergen Institute is the institute for economic research of the Erasmus Universiteit Rotterdam, Universiteit van Amsterdam, and Vrije Universiteit Amsterdam.

Tinbergen Institute Amsterdam

Roetersstraat 31

1018 WB Amsterdam

The Netherlands

Tel.: $\quad+31(0) 205513500$

Fax: $\quad+31(0) 205513555$

Tinbergen Institute Rotterdam

Burg. Oudlaan 50

3062 PA Rotterdam

The Netherlands

Tel.: $\quad+31(0) 104088900$

Fax: $\quad+31(0) 104089031$

Please send questions and/or remarks of nonscientific nature to driessen@tinbergen.nl.

Most TI discussion papers can be downloaded at http://www.tinbergen.nl. 


\title{
Measuring Synchronisation and Convergence of Business Cycles
}

\author{
Siem Jan Koopman* and João Valle e Azevedo \\ Department of Econometrics, Free University, NL-1081 HV Amsterdam \\ Tinbergen Institute, Amsterdam
}

June 17, 2003

\begin{abstract}
This paper investigates business cycle relations among different economies in the Euro area. Cyclical dynamics are explicitly modelled as part of a time series model. We introduce mechanisms that allow for increasing or diminishing phase shifts and for time-varying association patterns in different cycles. Standard Kalman filter techniques are used to estimate the parameters simultaneously by maximum likelihood. The empirical illustrations are based on gross domestic product (GDP) series of seven European countries which are compared with the GDP series of the Euro Area and that of the United States. The original integrated time series are band-pass filtered. We find that there is an increasing resemblance between the business cycle fluctuations of the European countries analysed and those of the Euro area, although with varying patterns.
\end{abstract}

Keywords: Band-pass filter; Cyclical convergence; Kalman filter; Unobserved components time series models; Phase shifts.

JEL classification: C13, C32, E32.

\footnotetext{
${ }^{*}$ Corresponding author: Department of Econometrics, Free University Amsterdam, De Boelelaan 1105, 1081 HV Amsterdam, The Netherlands. Telephone: +31 204446019 Fax: +31 204446020 Email: s.j.koopman@feweb.vu.nl. We would like to thank Marius Ooms and Gerhard Rünstler for discussions and comments. All remaining errors are ours.
} 


\section{Introduction}

The comparisons of business cycle comovement among different economies is usually static. Ad-hoc sub-samples are analysed by computing parametric and non-parametric statistics from which some conclusions are drawn. The explicit modelling of relevant features does not take place. In this paper we propose a modelling strategy that avoids many of the shortcomings of previous studies. Important features and relevant regime switches are estimated simultaneously within a multivariate time series model. Time-varying phase shifts and time-varying degrees of association are explicitly modelled. Transition to different regimes will take place smoothly.

The main motivation on developing these models is the analysis of business cycles relations within the European Union countries, particularly those in the Euro area. In a context of single currency and common monetary policies in the Euro area, the resemblance of the business cycles of the participant countries is a major concern. This paper also contributes to the documentation of the degree of association and synchronisation between the aggregate Euro Area output business cycle fluctuations and those of various European Union countries as well as the U.S. The results presented are mostly in line with those from similar studies. Namely, there is an increasing resemblance (higher degree of association and higher synchronisation) among the business cycles of the European Union countries analysed, although with some important exceptions. The purpose of our analysis is merely descriptive. There is no attempt to state that the main features of the recent past can be relevant in future analysis or judgement. Furthermore, we focus entirely on time series dynamics; no explanation for the identified stylised facts is attempted.

\section{$1.1 \quad$ Literature overview}

Various authors have tried to assess and explain business cycles convergence and synchronisation. Artis and Zhang (1997) address the question of whether the Exchange Rate Mechanism (ERM) has implied an increasing conformity among the business cycles of the participant countries. Two sub-samples are analysed, corresponding to the periods before and after the formation of the ERM. The Hodrick-Prescott (HP) filter is used to obtain the cyclical component of industrial production indices. They show that the cycles in the ERM countries became more synchronised with the German one. Angeloni and Dedola (1999) compare business cycle fluctuations of output, industrial production, stock indices and prices across countries in various sub-samples. These fluctuations are recovered using the HP filter but also 1-quarter and 4-quarters logarithmic differences. They conclude that in the latest sub-sample, from 1993 to 1997, correlations are almost always higher, suggesting the existence of encouraging signs regarding the success of the monetary union. Also, they add to the analysis the comparison of correlation between supply and demand shocks across countries in the same sub-samples. The 
shocks are estimated applying the bivariate structural vector autoregressive (VAR) methodology of Bayoumi and Eichengreen (1993). In this case the previous conclusion would not be valid, since no evidence was found on higher degrees of association of the identified shocks in the more recent period. Wynne and Koo (2000) document differences and similarities between business cycles in the European Union and business cycles in the Federal Reserve districts in the U.S. The business cycle fluctuations are recovered using the band-pass filter proposed by Baxter and King (1999). They compare business cycle fluctuations of output, employment and prices using simply linear correlation estimated by generalised method of moments. Belo (2001) uses several parametric and non-parametric statistics to investigate whether annual output cycles obtained with the HP filter have converged to the Euro area cycle. The analysis of convergence was made by considering two and sometimes three sub-samples. He concludes that there was in general an increase in the various measures of association employed, identifying the patterns documented by Wynne and Koo (2000). Additionally, he identifies a leading cycle from the U.S. and the U.K., when compared to the Euro area. However, if the time shifts in the cross-correlation functions are taken into account, then the U.K. displays a strong association with the Euro area in the period 1979-1999, whereas in the case of the U.S. this association is modest.

\subsection{Contribution of paper}

Our approach differs from common practice in important ways. For instance, instead of comparing parametric and non-parametric statistics in various sub-samples, we use an extension of the multivariate unobserved components model with phase shifts among cyclical components developed by Rünstler (2002). This model is itself an extension of the similar cycles model proposed by Harvey and Koopman (1997). We incorporate mechanisms that model either increasing or diminishing phase shifts as well as mechanisms that model time-varying association patterns in the cyclical components. Regime switches may appear as limiting cases, but the time points of transition are estimated, not imposed by the researcher. Since these extensions are only related to the cycle component of the model, usual trend specifications can also be considered. However, since our focus is on dynamic relations among different cycles, we will isolate these by imposing a definition of cyclical fluctuations. Specifically, the original integrated series will be band-pass filtered. We will however use the best performing and most flexible linear filter available, the band-pass filter proposed by Christiano and Fitzgerald (2003). This

means, just as in the case of the authors aforementioned, that our focus is on the growth cycle, the deviations of output from its trend. This follows the definition of business cycles by Lucas (1977). 
There has been a renewed interest in classical cycle analysis, which follows the seminal contribution of Burns and Mitchell (1946). However, instead of the more or less ad-hoc dating of the peaks and troughs in the level of various series, both univariate and multivariate Markovswitching (vector) autoregressions (MS-VAR) have been employed, following extensions of the model proposed by Hamilton (1989). Diebold and Rudebusch (1996) incorporate dynamic factors in these models, allowing the simultaneous analysis of comovement and regime switch. This implicitly means the imposition of restrictions in the VAR representation of the model. However, their intention was mainly to capture comovements across many variables in the same economy, not across economies. Krolzig and Toro (2002) show that in the analysis of European GDP series the MS-VAR models capture dynamics that are consistent with the classical approach. The analysis of comovement and synchronization within these models (either univariate or multivariate) can be made using non-parametric statistics such as concordance (see Harding and Pagan (1999)), by simply comparing dates at which the various regimes coincide or by looking at contemporaneous correlation of the smoothed probability of being in a recession (see Artis, Krolzig, and Toro (2002)). Other stylized facts can be detected such as the differences in volatility and duration of each regime. We view our approach as complementary to this. Our focus is on business cycle frequencies, or frequencies usually associated with the business cycle. The detrending and modeling strategy implies straightforward interpretations of the proposed measures of association and synchronisation. In the above approaches, the assessment of at least synchronisation is judgmental. It may mask differences in the dynamics of fluctuations at usual business cycle frequencies. Coincidence in the regime is not sufficient (not even necessary) to coincidence phases in periodic components at different frequencies.

The empirical results presented are consistent with those of previous studies. For example, France and Germany display a high degree of association with the Euro area across the sample. Their cycles are synchronised with those of the Euro area. Spain and Italy became more synchronised with the Euro area in the last twenty years. The U.S. leading cycle with respect to the Euro area and the lower degree of association are well established in the literature and are also identified by our model.

\subsection{Plan of paper}

The remaining of the paper is organised as follows. In section 2 the similar cycles model with shifts is presented. In section 3 this model is extended to include time-varying phase shifts and time-varying covariance matrices. In section 4 estimation results, obtained by considering bivariate combinations of real GDP series from various economies, are presented. Section 5 concludes. 


\section{Common similar stochastic cycles with shifts}

This section introduces the multivariate unobserved component model with stochastic cycles. The cycles can be subject to shifts and can be correlated, contemporaneously and between different time periods. Limiting cases include cycles that evolve independent of each other over time and cycles that are common to each other. The development of the models in this section are discussed in detail in Appendices B and $\mathrm{C}$ for univariate and bivariate versions of the general multivariate model. Some basics on trigonometrics and cycles are provided in Appendix A.

\subsection{Multivariate stochastic cycle model}

Various specifications for the multivariate cycle component $\psi_{t}$ can be considered in general. For example, multiple cycles can be modelled within vector autoregressive processes, see Chan and Wallis (1978) and Bayoumi and Eichengreen (1993) for illustrations. In this paper we model multivariate stochastic cyclical processes explicitly by trigonometric terms with time-varying amplitudes and phases. Such stochastic cyclical components can be embedded in a multivariate unobserved components time series model. The stochastic cycle vector $\psi_{t}$ will be modelled as

$$
\left(\begin{array}{c}
\psi_{t+1} \\
\psi_{t+1}^{+}
\end{array}\right)=\phi\left[\begin{array}{cc}
\cos (\lambda) I_{N} & \sin (\lambda) I_{N} \\
-\sin (\lambda) I_{N} & \cos (\lambda) I_{N}
\end{array}\right]\left(\begin{array}{c}
\psi_{t} \\
\psi_{t}^{+}
\end{array}\right)+\left(\begin{array}{c}
\kappa_{t} \\
\kappa_{t}^{+}
\end{array}\right), \quad t=1, \ldots, n
$$

where $I_{k}$ is the $k \times k$ identity matrix. The $N \times 1$ vector $\psi_{t}$ consists of similar cycles with common frequency $\lambda$ and common autoregressive coefficient $|\phi|<1$. The $N \times 1$ vector $\psi_{t}^{+}$is a constructed variable; see Appendices $\mathrm{A}$ and $\mathrm{B}$. The $N \times 1$ disturbance vectors $\kappa_{t}$ and $\kappa_{t}^{+}$are serially and mutually uncorrelated, and are normally distributed with mean zero and variance matrix

$$
\operatorname{Var}\left(\begin{array}{c}
\kappa_{t} \\
\kappa_{t}^{+}
\end{array}\right)=\left[\begin{array}{cc}
\Sigma_{\kappa} & 0 \\
0 & \Sigma_{\kappa}
\end{array}\right]
$$

which implies that $\kappa_{t}$ and $\kappa_{t}^{+}$have a common variance matrix $\Sigma_{\kappa}$. This specification generates stationary multiple cyclical processes with period $f=2 \pi / \lambda$. The individual cycles in $\psi_{t}$ have similar properties due to the common $\phi$ and $\lambda$. Similar cycles have been introduced by Harvey and Koopman (1997) and they have explored the stochastic properties. For example, the autocovariance function for $\psi_{t}$ is given by

$$
\Gamma(\tau)=\left(1-\phi^{2}\right)^{-1} \phi^{\tau} \cos (\tau \lambda) \Sigma_{\kappa}, \quad \tau=0,1,2, \ldots
$$

from which it follows that the variance matrix of the cycle is given by $\Gamma(0)=\left(1-\phi^{2}\right)^{-1} \Sigma_{\kappa}$. The imposition of the same period of the cycle and the same damping factor is appealing, as it reflects the similar dynamics of business cycle fluctuations across macroeconomic time series 
from the same economy or from different economies. In the case of our empirical study in section 4, there may be evidence that the persistence of real GDP business cycle fluctuations varies across countries, the differences are not dramatic, see also Azevedo (2002).

The vector variable $y$ that is subject to a cyclical time series process and that is possibly measured with noise can be modelled by

$$
y_{t}=\mu+\psi_{t}+\varepsilon_{t}, \quad \varepsilon_{t} \sim \mathcal{N} \mathcal{I D}\left(0, \Sigma_{\varepsilon}\right), \quad t=1, \ldots, n
$$

where $y_{t}$ is a $N \times 1$ vector of time series. The $N \times 1$ constant vector $\mu$ is unknown and the $N \times 1$ irregular vector $\varepsilon_{t}$ with mean zero and $N \times N$ variance matrix $\Sigma_{\varepsilon}$ is mutually uncorrelated with the disturbances associated with the cycle vector $\psi_{t}$, both contemporaneously and between different time periods. The unknown parameters in the cycle model (2) are the variance matrices $\Sigma_{\varepsilon}$ and $\Sigma_{\kappa}$ together with the autoregressive coefficient $\phi$ and the cycle frequency $\lambda$. These parameters can be estimated by maximum likelihood for which the Kalman filter is employed to compute the loglikelihood function for a given set of parameters, see Harvey (1989) and Durbin and Koopman (2001).

For the purpose of estimation, parameters are transformed into a vector $\theta$ such that the likelihood maximation process can take place without constraints. The irregular variance matrix is assumed diagonal and specified as

$$
\Sigma_{\varepsilon}=\operatorname{diag}\left\{\exp \left(2 \theta_{\varepsilon, 1}\right), \ldots, \exp \left(2 \theta_{\varepsilon, N}\right)\right\},
$$

where $\theta_{\varepsilon, i}$ is the $\log$ standard deviation of the $i$-th element of irregular vector $\varepsilon_{t}$ for $i=1, \ldots, N$. The cycle disturbance variance matrix is specified as

$$
\Sigma_{\kappa}=C R C,
$$

where matrix $C$ is diagonal with standard deviations and matrix $R$ consists of contemporaneous correlations, that is

$$
C=\operatorname{diag}\left\{\exp \left(\theta_{\kappa, 1}\right), \ldots, \exp \left(\theta_{\kappa, N}\right)\right\}, \quad R=\left[\begin{array}{cccc}
1 & \rho_{\kappa, 2,1} & \cdots & \rho_{\kappa, N, 1} \\
\rho_{\kappa, 2,1} & 1 & \cdots & \rho_{\kappa, N, 2} \\
\vdots & \vdots & \ddots & \vdots \\
\rho_{\kappa, N, 1} & \rho_{\kappa, N, 2} & \cdots & 1
\end{array}\right],
$$

where $\theta_{\kappa, i}$ is a log standard deviation and matrix $R$ is restricted to be positive semi-definite. For the bivariate case of $N=2$ it is sufficient to have $-1 \leq \rho_{\kappa, 2,1} \leq 1$ which we specify as

$$
\rho_{\kappa, 2,1}=\theta_{\kappa, 2,1} / \sqrt{1+\theta_{\kappa, 2,1}^{2}},
$$

so that $\rho_{\kappa, 2,1} \rightarrow \pm 1$ as $\theta_{\kappa, 2,1} \rightarrow \pm \infty$. When correlations are estimated zero (or significantly close to zero), the individual cycles in $\psi_{t}$ are independent of each other. In the opposite case, 
when correlations are significantly close to one (that is \pm 1 ), individual cycles are common and variance matrix $\Sigma_{\kappa}$ has a lower rank. For the multivariate case $N>2$, it is more intricate to specify $R$ subject to the appropriate restrictions. A possible specification is

$$
R=K^{-1} L L^{\prime} K^{-1}, \quad K=\left[\operatorname{diagonal}\left(L L^{\prime}\right)\right]^{1 / 2}
$$

where $L$ is a lower unity triangular matrix and $\operatorname{diagonal}(A)$ is a diagonal matrix with the corresponding diagonal elements of matrix $A$. The transformation ensures that $R$ has the interpretation of a correlation matrix and that it is always positive semi-definite. The lower triangular elements of matrix $L$ can be estimated without further restrictions. It turns out that the specification of $R$ in (6) for $N=2$ is equivalent to (5). A large negative or positive element in the lower triangular part of $L$ implies that the corresponding element in $R$ is close to one or minus one, respectively. Finally, we have $\phi=\exp \theta_{\phi} /\left(1+\exp \theta_{\phi}\right)$ and $f=2+\exp \theta_{\lambda}$ where $\theta_{\phi}$ and $\theta_{\lambda}$ are the transformed parameters that can be estimated directly for the cycle model.

\subsection{Multivariate stochastic cycle model with shifts}

The multivariate cycle model can be modified to allow for phase shifts between cycles as is shown by Rünstler (2002). A time series model for a vector variable $y$ with a multivariate stochastic cycle component that can be subject to shifts is given by

$$
y_{t}=\mu+\operatorname{diag}\left\{\cos \left(\lambda d_{\xi}\right)\right\} \psi_{t}+\operatorname{diag}\left\{\sin \left(\lambda d_{\xi}\right)\right\} \psi_{t}^{+}+\varepsilon_{t}
$$

where $d_{\xi}$ is the real vector

$$
d_{\xi}=\left(\xi_{1}, \ldots, \xi_{N}\right)^{\prime}
$$

with its first element restricted to be equal to zero, that is $\xi_{1}=0$. We notice that

$$
\begin{aligned}
\operatorname{diag}\left\{\cos \left(\lambda d_{\xi}\right)\right\} & =\operatorname{diag}\left\{1, \cos \left(\lambda \xi_{2}\right), \ldots, \cos \left(\lambda \xi_{N}\right)\right\} \\
\operatorname{diag}\left\{\sin \left(\lambda d_{\xi}\right)\right\} & =\operatorname{diag}\left\{0, \sin \left(\lambda \xi_{2}\right), \ldots, \sin \left(\lambda \xi_{N}\right)\right\}
\end{aligned}
$$

where $\operatorname{diag}\{x\}$ is a diagonal matrix with its diagonal elements as in vector $x$. The restriction implies that the phase of the first cycle element in $\psi_{t}$ is the reference for the phase shifts of the other cycle elements. The phase shift between $y_{i t}$ and $y_{j t}$ is given by $\xi_{j}-\xi_{i}$ for $i, j=1, \ldots, N$. The variance of the cycle component is given by

$$
\Gamma(0)=\frac{1}{1-\phi^{2}} \Sigma_{\kappa} \odot \cos (\Lambda), \quad \Lambda=\lambda\left(\mathbf{1} d_{\xi}^{\prime}-d_{\xi} \mathbf{1}^{\prime}\right),
$$

and the (multivarariate) autocovariance function is given by

$$
\Gamma(\tau)=\frac{\phi^{|\tau|}}{1-\phi^{2}} \Sigma_{\kappa} \odot \cos \left(\Lambda_{\tau}\right), \quad \Lambda_{\tau}=\lambda\left(\tau \mathbf{1 1} \mathbf{1}^{\prime}+\mathbf{1} d_{\xi}^{\prime}-d_{\xi} \mathbf{1}^{\prime}\right)
$$


where 1 is the unity vector $(1, \ldots, 1)^{\prime}$ and where $\cos (\lambda A)$ is a matrix with its element $(i, j)$ equal to $\cos \left(\lambda A_{i j}\right)$ for any real matrix $A$ with $A_{i j}$ as its $(i, j)$ element. We note that $\odot$ refers to element by element multiplication so that the $(i, j)$ element of $C=A \odot B$ is $C_{i j}=A_{i j} B_{i j}$ for all elements.

Proof of variance and autocovariances is given by Rünstler (2002, Appendix A.1). When $\Sigma_{\kappa}$ is diagonal, $\Gamma(\tau)$ is also diagonal and does not depend on vector $d_{\xi}$ since the leading diagonal of matrix $d_{\xi} \mathbf{1}^{\prime}-\mathbf{1} d_{\xi}^{\prime}$ is zero. In this case phase shifts are not identifiable. For estimation purposes the shift element $\xi_{i}$ is transformed as

$$
\xi_{i}=\frac{\pi}{\lambda}\left[\exp \theta_{\xi, i}\left(1+\exp \theta_{\xi, i}\right)^{-1}-0.5\right]
$$

that ensures $-\pi / 2<\lambda \xi_{i}<\pi / 2$ for $i=2, \ldots, N$. Also, $\theta_{\xi, i}=0$ implies $\xi_{i}=0$.

Phase shifts are therefore modelled as shifts in the autocovariance function of the cycle component itself. This means that the effect of (correlated) innovations in the behaviour of the cycle component might be subject to time shifts when compared to model (1), but with the same dynamical behaviour. This property allows us to define the correlation matrix implied by $\Sigma_{\kappa}$ in (9), as the phase adjusted correlation matrix among cyclical innovations, which is also the phase adjusted correlation matrix among cyclical elements. Also from (9) the autocovariances of a particular series are opportunely independent of the phase shifts. It is also clear that phase shifts are transitive across series. Finally, restriction (10) implies no ambiguity in the definition of pro or counter cyclicality. As shown by Rünstler (2002), phase shifts as defined above and the corresponding phase adjusted correlation matrix are closely related to coherence and phase spectra of model (7), respectively. However, in the remaining of the paper we will only consider the time domain interpretations. Some further consequences of a bivariate cycle component with a shift are discussed in Appendix C.

\section{Synchronisation and convergence of cycles}

\subsection{Synchronisation of multiple cycles}

The multivariate model (7) with a similar stochastic cycle component with shifts can be modified to have different shift lengths at different periods. For instance, the shift between two variables can be relatively large in one period and relatively small in another period. It is particularly interesting to investigate whether business cycles of, for example, European countries are in the process of synchronisation. This implies that the cyclical processes of two business cycle components are shifted from each other by a small number of time-points in earlier years while they match (without shifts) in more recent years. The time-varying nature of the shift parameter $\xi_{i}(i=2, \ldots, N)$ in model $(7)$ can be established in different ways. Firstly, different 
$\xi_{i}$ 's for different time periods can be considered so that shifts over time are subject to structural breaks. The periods with different shifts need to be selected a-priori and this needs to be done on an ad-hoc basis. Secondly, the shift can be taken as a flexible but smooth function depending on time. For example, a regression spline function can be considered as is described by Poirier (1976). Also in this case some degree of ad-hoc decisions need to be made a-priori to control for flexibility at the cost of smoothness.

In the remainder of this paper we will specify the time-variation of a phase shift via the deterministic logit function

$$
\xi_{i, t}=\frac{\pi}{\lambda}\left\{\exp \theta_{\xi_{i}}\left(1+\exp \theta_{\xi_{i}}\right)^{-1}-0.5\right\} \times \exp \left(s_{\xi_{i}, t}\right)\left\{1+\exp \left(s_{\xi_{i}, t}\right)\right\}^{-1}, \quad s_{\xi_{i}, t}=s_{\xi_{i}} \times\left(t-\tau_{\xi_{i}}\right),
$$

for $i=2, \ldots, N$ and where $s_{\xi_{i}}$ determines the shape of the logit function and $\tau_{\xi_{i}}$ determines the mid-time position of the change. The parameters $\theta_{\xi_{i}}, s_{\xi_{i}}$ and $\tau_{\xi_{i}}$ can be estimated within the maximum likelihood process provided that correlations between the cycle exist (non-zero); see the discussion in Appendix C. This specification allows for the estimation of $\tau_{\xi_{i}}$ which implies that no a-priori decision is needed about the time when a change in the shift occur. The limitation of our specification of cycle synchronisation (11) is that the logit function is monotone in time. The function either increases or decreases depending on $\theta_{\xi_{i}}$. For the purpose of our empirical study in the next section, the limitation is useful since we want to determine whether business cycles of European countries do synchronise or do not synchronise. Similar logit mechanisms as in (11) have been used in the context of nonlinear smooth transition autoregressive models which are reviewed in van Dijk, Terasvirta, and Franses (2002).

The consequence of modelling synchronisation is that the dynamic properties of the cycle component is not time-invariant. In the same way the shift depends on a deterministic function of time, the autocovariance function (9) also depends on time since $\Lambda_{\tau}$ is time-varying, that is

$$
\Lambda_{\tau, t}=\lambda\left(\tau \mathbf{1} \mathbf{1}^{\prime}+\mathbf{1} d_{\xi, t}^{\prime}-d_{\xi, t} \mathbf{1}^{\prime}\right), \quad d_{\xi, t}=\left(0, \xi_{2, t}, \ldots, \xi_{N, t}\right)^{\prime}
$$

A similar type of time-variation applies to variance matrix $\Gamma(0)$ in $(8)$.

\subsection{Convergence for multiple stochastic cycles}

The multivariate stochastic cycle model (2) or the model with shifts (7) can be modified so that correlations between the cycle elements are not constant for all time points $t=1, \ldots, n$. For instance, we may have two distinct periods for which in the first period cycles are correlated, that is $\left|\rho_{\kappa, i, j}\right|<1$ whereas in the other period the cycles are common and, therefore, perfectly correlated, that is $\rho_{\kappa, i, j}=1$. This case is of interest from an economic perspective since we can refer to these as converging cycles. It implies that comovements between cycles become stronger and can even become equivalent (up to a scaling constant). An interesting economic example 
is the consideration of business cycles of, say, Germany and France which may be moving more closely due to the process of economic and monetary integration in Europe.

To allow for a smooth transition from one period to another period with different correlations between two similar cycles, we follow a similar strategy as in Luginbuhl and Koopman (2003) although a different specification is used in this paper. The variance matrix of the cycle disturbance vector $\Sigma_{\kappa}$ can be made time-varying by incorporating smooth time functions within the decomposition of the correlation matrix $R$ in (6). By adopting the variance specification in (3), the correlations can be made time-varying directly while the variances remain constant over time. The correlations are made time-varying via a smooth time function (similar to the one used for synchronisation) for a particular lower triangular element of $L$ in (6). In the bivariate case, the correlation between the two elements of the cycle disturbance vector is specified as

$$
\rho_{\kappa, 2,1}= \pm\left[1-(1-b) \times \exp \left(s_{\kappa_{2}, t}\right)\left\{1+\exp \left(s_{\kappa_{2}, t}\right)\right\}^{-1}\right], \quad s_{\kappa, 2,1, t}=s_{\kappa, 2,1} \times\left(t-\tau_{\kappa, 2,1}\right),
$$

for $i, j=1, \ldots, N, i \neq j$, where coefficient $s_{\kappa, i, j}$ determines the shape of the function and $\tau_{\kappa, i, j}$ the midtime-point at which the transition takes place. The logit function keeps correlation values between zero and one. The coefficient $b$, which should be between zero and one, adds further flexibility and it ensures that the correlation is between $b$ and one. For the purpose of estimation $b$ is specified as $b=\left[1+\exp \left(\theta_{b}\right)\right]^{-1}$ where $\theta_{b}$ is an unknown coefficient. The specification in (12) allows the time-varying correlations to be positive or negative only. This restriction can be relaxed but for the illustrations in the next section this has proven to be a natural restriction. The coefficients $s_{\kappa, i, j}, \tau_{\kappa, i, j}$ and $\theta_{b}$ can be estimated simultaneously within the likelihood maximisation procedure. Finally, an alternative is to adopt the dynamic conditional correlation specification of Engle (2002) for a time-varying correlation matrix $R$.

\section{Bivariate illustrations of GDP for Euro area and U.S.}

\subsection{Data transformations}

To study the properties and relations among business cycles fluctuations of real GDP using the models presented in the previous section we need first to define and measure the business cycle. We follow Baxter and King (1999) who define business cycles as "fluctuations with a specified range of periodicities". We define this range as the 6 to 32 quarters band, the band usually considered in the analysis of the U.S. and Euro area business cycles; see Stock and Watson (1998) for the U.S. and the ECB (2001) report for the Euro area. It follows that fluctuations with periodicities below 6 quarters are considered noisy and consequently not important to business cycle dynamics and periodicities above 32 quarters are part of a slowly evolving component related to long-term growth. We do not provide an economic interpretation 
of the fluctuations. All we want is to analyse components that produce specified deviations from an apparently ever-growing component. An important element of definition is assumed in this approach.

By defining business cycles as fluctuations within a range of periodicities (corresponding to a range of frequencies in the frequency domain) the ideal filter should retain the desired frequencies and perfectly eliminate the remaining while inducing no phase shift. Since it is not possible in a finite sample to apply an ideal filter we use the approximation proposed by Christiano and Fitzgerald (2003). This filter is obtained by minimising, for each $t$, the function $Q$ with respect to the filter weights where

$$
Q=\int_{-\pi}^{\pi}\left|W(\omega)-B_{t}\left(e^{-i \omega}\right)\right|^{2} f_{x}(\omega) d \omega
$$

with $W(\omega)$ as the frequency response function of the ideal filter isolating the desired frequencies band. The frequency response function $B_{t}\left(e^{-i \omega}\right)$ is for the filter

$$
B_{t}(L)=\sum_{j=-(T-t)}^{t-1} b_{t, j} L^{j},
$$

with sample size $T$, lag operator $L\left(L^{ \pm s} y_{t}=y_{t \mp s}\right)$ and filter weights $b_{t, j}$. We note that $e^{-i \omega}=$ $\cos \omega-i \sin \omega$ with $i=\sqrt{-1}$. Finally, $f_{x}(\omega)$ in (13) is the spectrum (or pseudo-spectrum, in the case of integrated series) of the observation series $x_{t}$ to be filtered.

This method of band-pass filtering is equivalent, in the time domain, to the method of obtaining optimal estimates (in the mean square error sense) of ideal filtered observations. The criterion $Q$ depends on the true process generating the data. But for unit-root series, Christiano and Fitzgerald (2003) show that the procedure is nearly optimal when the process is assumed to follow a random walk. There is little advantage in knowing (or estimating) the process. We will therefore use this nearly optimal approximation.

We could use the models proposed by Harvey and Koopman (1997) to decompose with a single model the trend and cycle components of real GDP series for various countries. However, this is not always a good option. Although business cycle dynamics are apparently similar across countries when we look at real GDP, the model may have difficulty in capturing those dynamics for some of them. This is a consequence of the dominant role played by low frequencies in the behavior of macroeconomic time series. These frequencies frequently mask business cycle dynamics. Also, the number of parameters would increase dramatically and make the estimation of the full model computationally burdensome. Another option would be to impose restrictions on a model with a trend specification in order to implicitly define a band-pass filter, see Harvey and Trimbur (2003). 
One of the consequences of using a band-pass filter is that the period of the cycle is roughly fixed. This period corresponds to the lower cut-off frequency chosen to define the band of interest. The advantage is that we do not need to estimate the period of the cycle and this will be equal for all series. However, the definition of business cycle fluctuations may be viewed as arbitrary. Still, for the purpose of this paper we are only interested in those fluctuations, even if they do not correspond to some ideal definition of the business cycle.

A slight drawback in the application of the referred filter is that the resulting series is not "exactly" stationary since the variance of filtered observations is time-varying. Also, there is some revision in the endpoints of the sample when new observations are available. Despite this, endpoints filtered observations are highly correlated with ideal filtered observations and the variance stabilises quite rapidly; see Azevedo (2003) for further details. Since these observations are informative we will not drop them.

\subsection{Estimation and computational details}

In the illustrations below we consider the multivariate stochastic cycle model (7) with timevarying shifts (11) and time-varying correlations (12). The former aims at detecting synchronisation and the latter aims at convergence. The implied covariance structure of the time series is time-varying due to these two sources of time variation: synchronization implies that matrix $\Lambda_{\tau}$ is time-varying and convergence implies that matrix $\Sigma_{\kappa}$ is time-varying. The autocovariance matrix $\Gamma(\tau)$ depends on both matrices and is therefore time-varying as well. Both mechanisms can be incorporated simultaneously and estimation can be carried out using standard Kalman filtering techniques. The cycle model can be casted straightforwardly into the linear Gaussian state space model that has time-varying system elements. State space methods are used for estimation and signal extraction; for a general and recent exposition see, for example, Durbin and Koopman (2001). The computations are implemented using the object-oriented matrix programming environment Ox 3.0 of Doornik (2001) and using the library of C functions for state space models SsfPack by Koopman, Shephard, and Doornik (1999). It is evident that estimation by numerically maximising the likelihood function becomes more problematic as the number of parameter increases while the number of observations remain constant.

Since we use band-pass-filtered data, noisy fluctuations have been eliminated. This means that the irregular component in (7) may be safely dropped ${ }^{1}$. This simplification is convenient. All the time-varying covariance matrices and shifts refer to the isolated cyclical fluctuations, not to a general unobservable cyclical component. Also, by definition, the mean of the bandpass filtered series is zero, so we fix $\mu=0$ in model (7). Additionally, the definition of cyclical fluctuations implies in general a period of the cycle of 32 quarters, so we fix $\lambda=2 \pi / 32$.

\footnotetext{
${ }^{1}$ In practice we estimate the model with the irregular variance set at $10^{-12}$ only to achieve numerical stability.
} 


\subsection{Empirical results for the Euro area}

We consider band-pass filtered time series of (logs of) real, seasonally adjusted GDP for six European Union countries (Germany, France, Italy, Spain, The Netherlands and the U.K.) and also for the Euro area (12 countries) aggregate and the U.S ${ }^{2}$. The sample consists of 32 years of quarterly observations and covers the periods 1970:1 to 2001:1. We estimate the model as described above with $N=2$, applying it to bivariate combinations of GDP series for the Euro area and the individual European countries. In addition we consider some bivariate combinations that involve GDP series of the U.S.

We first report and discuss estimation results for the GDP series of six European countries individually that are modelled together with the GDP series of the Euro area. All figures in this section display the estimated time-varying phase shift, time-varying phase adjusted correlation, time-varying contemporaneous correlation and the similar cycle components for the GDP series of the Euro area and an individual country.
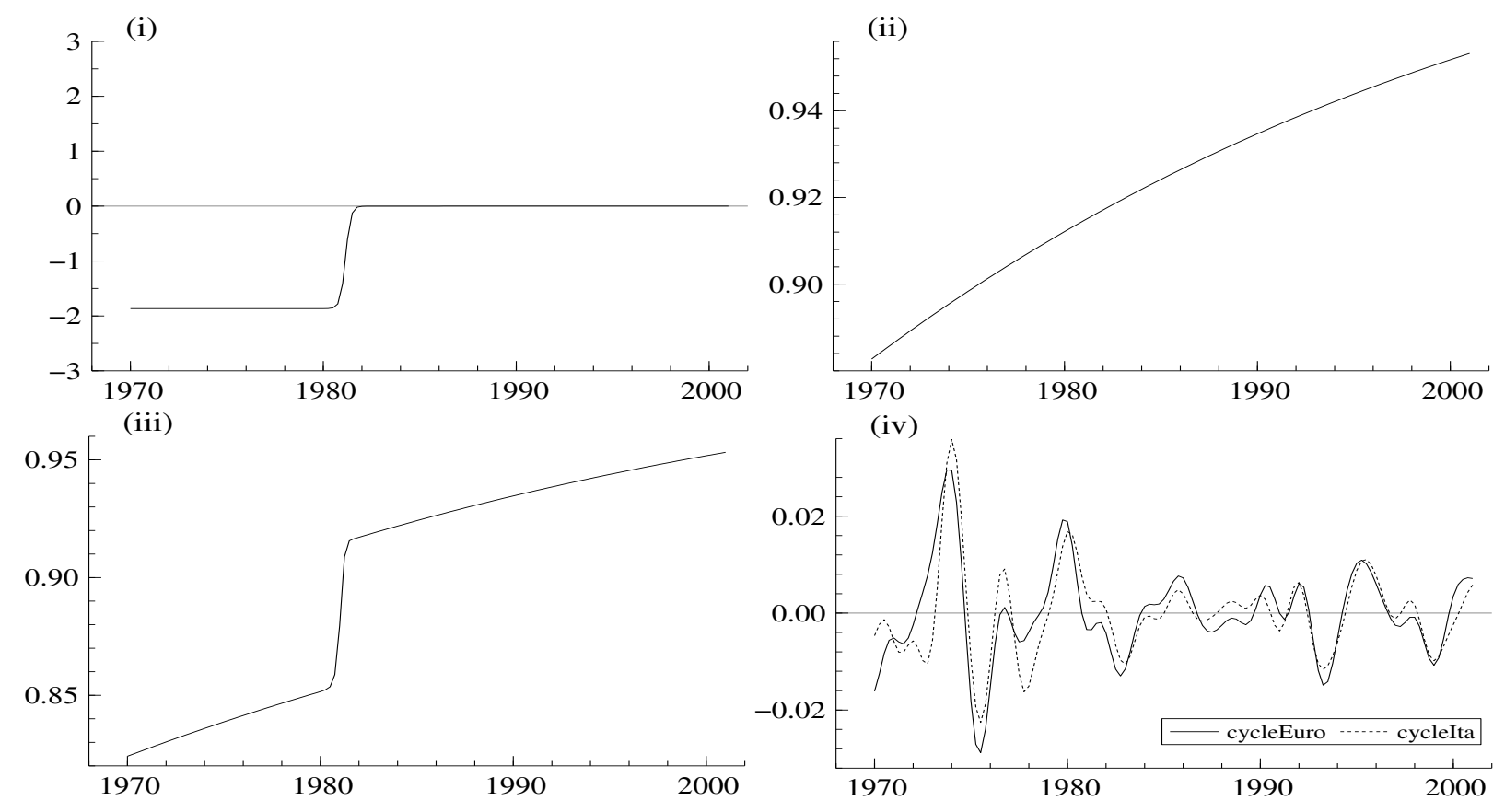

Figure 1: Estimated cycle model for Italy and Euro area: (i) estimated time-varying phase shift;

(ii) time-varying phase adjusted correlation; (iii) time-varying contemporaneous correlation; (iv)band-pass filtered series (rescaled)

Figure 1 compares the Euro area cycles to those of Italy. It can be seen that the model detects a phase shift of around two quarters in the 1970's, with Italy lagging the Euro area

\footnotetext{
${ }^{2}$ The data for individual countries is obtained from OECD Main Economic indicators. For the Euro area data is obtained from Fagan, Henry, and Mestre (2001).
} 
cycle. From 1980 onwards the estimated phase shift is zero. The phase-adjusted correlation is around 0.88 in 1970 and it steadily converges to almost 1. Contemporaneous correlation is naturally smaller in 1970 (around 0.8) and it displays a jump in 1980, coinciding with the break in the phase effect at that period.

Germany leads the Euro area cycle by more than two quarters in the early 1970's. This phase shift then decreases sharply, reaching zero around 1982. Phase adjusted correlation is around 0.83 until around 1985. It then increases steadily, converging to almost 1 . Contemporaneous correlation is around 0.75 in the 1970's. It then displays a jump around 1982 and thereafter it steadily increases, but with higher rates in the 1990's.
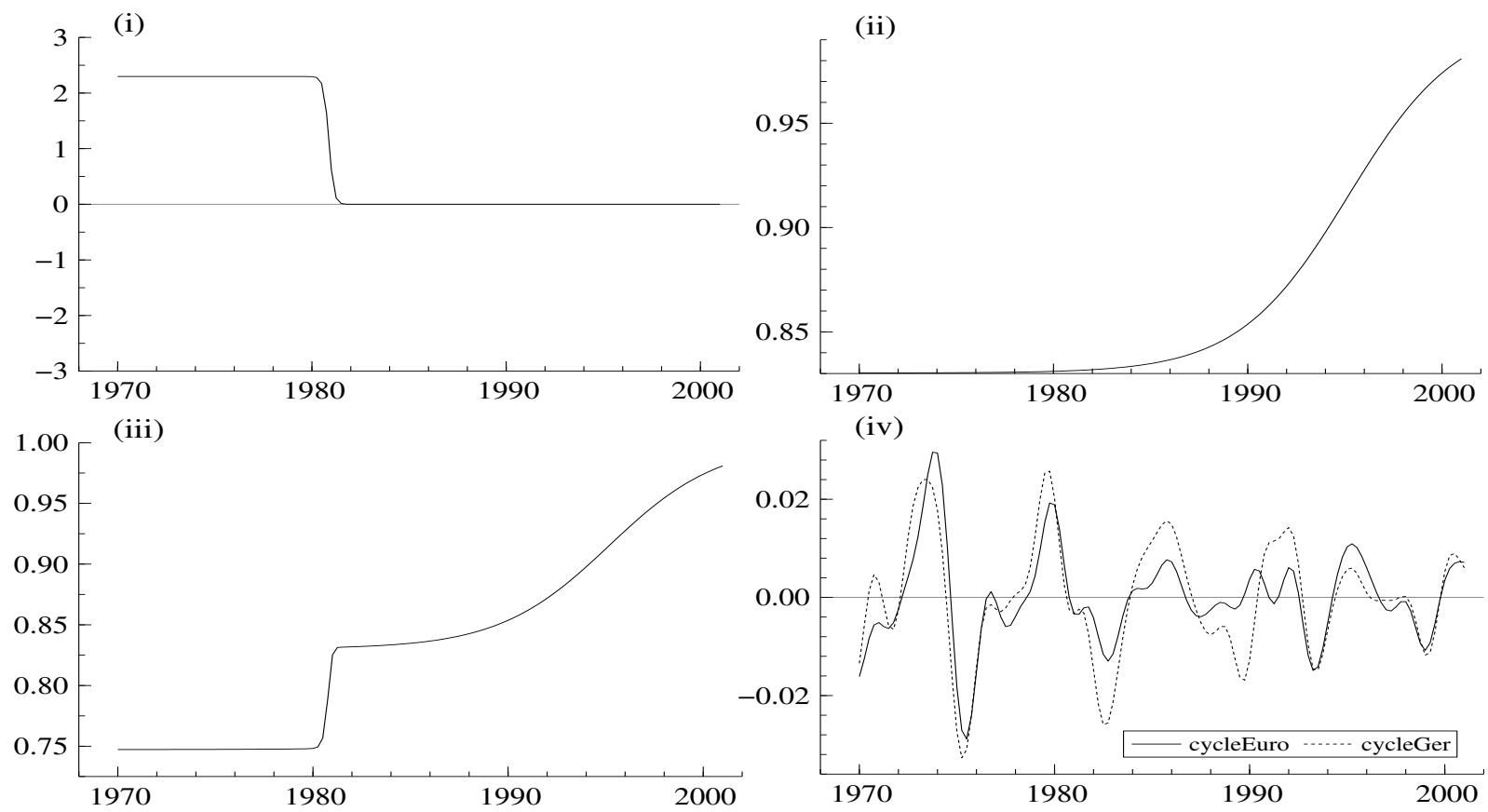

Figure 2: Estimated cycle model for Germany and Euro area: (i) estimated time-varying phase shift; (ii) time-varying phase adjusted correlation; (iii) time-varying contemporaneous correlation; (iv) band-pass filtered series (rescaled)

For France the phase effect is negligible in the whole sample. The estimated model implies a very small lead of the Euro area in the 1970's, not even reaching one quarter. Phase adjusted correlation is 0.83 in 1970 and it then increases steadily, reaching more than 0.95 in the end of the sample. Given the small estimated phase shift, contemporaneous correlation equals phase-adjusted correlation, the only exception (not relevant) occurring in the 1970's.

Comparing business cycle fluctuations of the Euro area to those of The Netherlands, we identify a zero phase effect until around 1986. From then on, The Netherlands displays a lead of around one quarter. Phase-adjusted correlation and contemporaneous correlation start with 

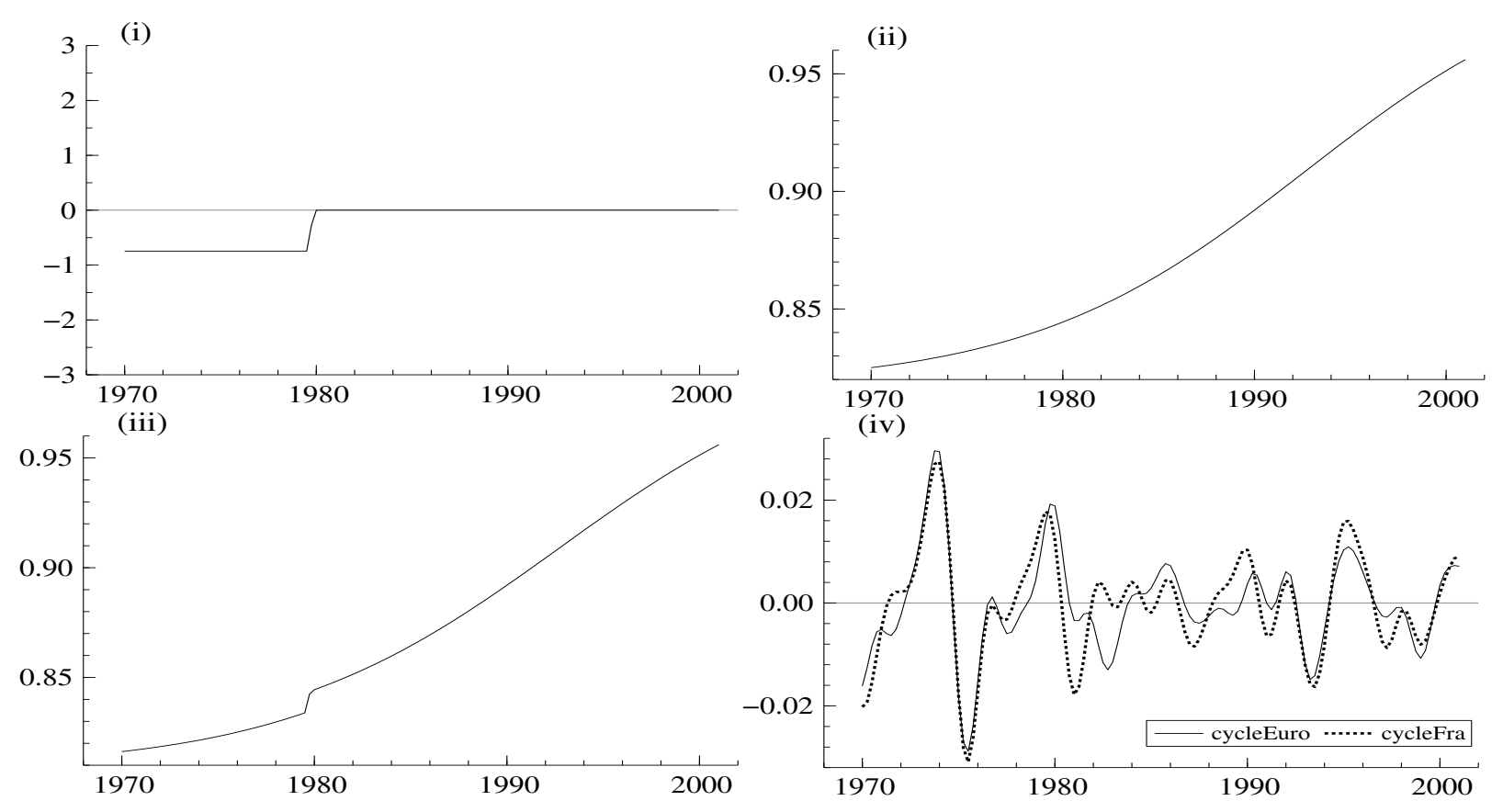

Figure 3: Estimated cycle model for France and Euro area: (i) estimated time-varying phase shift; (ii) time-varying phase adjusted correlation; (iii) time-varying contemporaneous correlation; (iv) band-pass filtered series (rescaled)

values below 0.6 , but increase steadily to values around 0.95 and 0.9 , respectively.

In the case of Spain, a lag of three quarters with relation to the Euro area is estimated in the period from 1970 to the early 1980's. The phase effect is estimated as zero thereafter. Both contemporaneous and phase adjusted correlation are, in the beginning of the sample, much lower than in the previous cases, starting respectively at 0.5 and 0.56 . A relevant increase in these measures of association occurs only during the 1990's.

Relevant increases in the overall correlation appear in most countries at the beginning of the 1980's, shortly after the start of the Exchange Rate Mechanism in 1979. However, further evidence would be needed to conclude that this event was the cause of the reported results.

\subsection{Empirical results for U.K., U.S. and Euro area}

The U.K. leads the Euro area by a bit more than three quarters in most of the sample. Only at the end of the sample the phase shift is estimated as zero. Phase adjusted correlation and contemporaneous correlation are also much lower than in previous cases but an increase in these measures of association takes place, reaching almost 1 in the end of the sample. This increased synchronisation and association of the U.K. with the Euro area in the last 5 to 6 years were not reported so far, also because data was not available. 

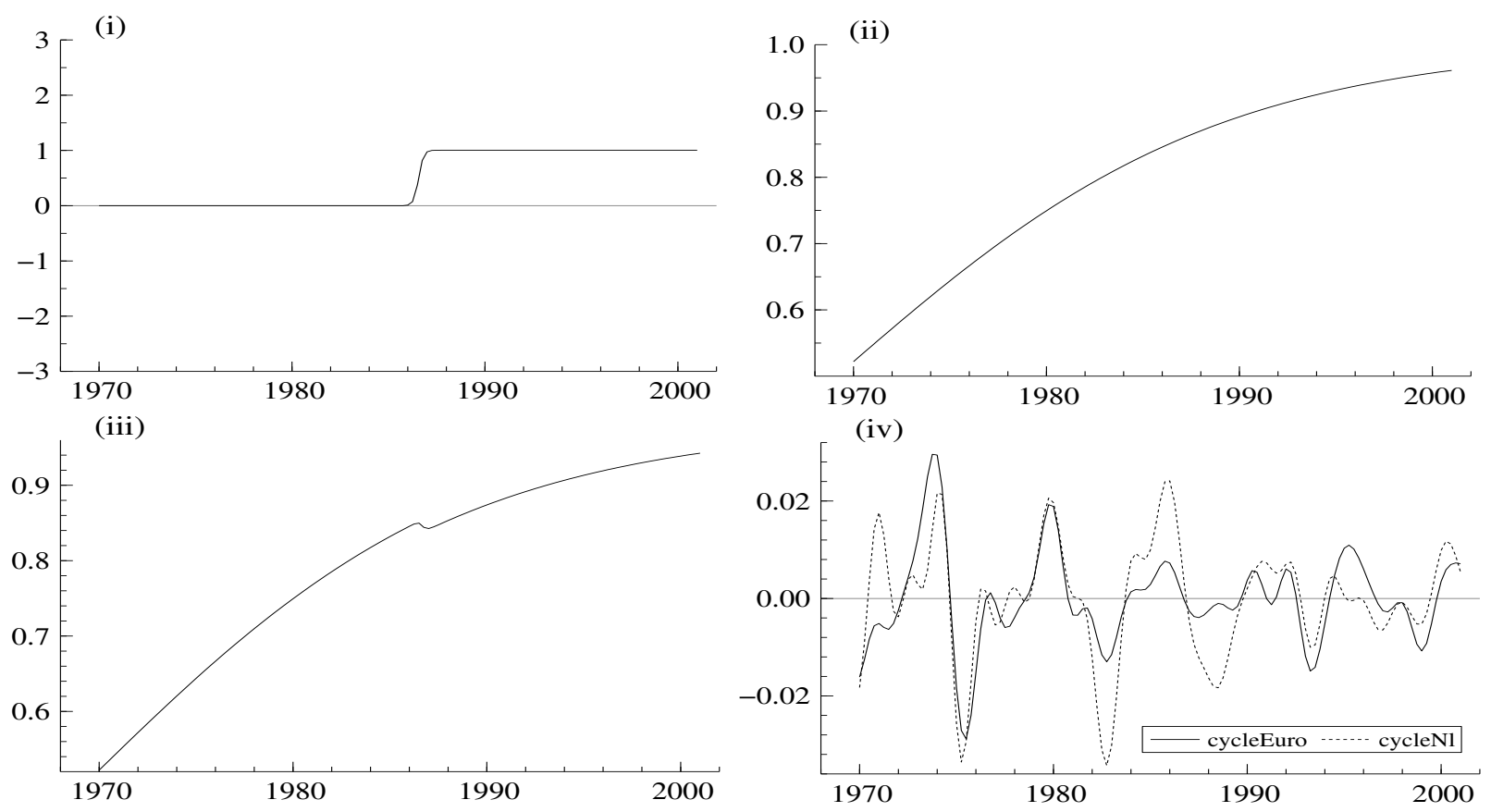

Figure 4: Estimated cycle model for the Netherlands and Euro area: (i) estimated time-varying phase shift; (ii) time-varying phase adjusted correlation; (iii) time-varying contemporaneous correlation; (iv) band-pass filtered series (rescaled)

With respect to the U.S., a lead of three quarters compared to Euro area is estimated in most parts of the sample. Only until 1972 the phase effect is estimated as zero. Phaseadjusted correlation increases steadily from around 0.4 in 1970 to 0.8 in 2000. Contemporaneous correlation varies between 0.4 and 0.7 , with a small decrease in the mid 1970's. These values for contemporaneous and phase-adjusted correlation in the 1970's are strikingly low and seem not to be consistent with a casual inspection of filtered series. This is also true when we compare the Euro area with the U.K.

It should be noted that the proposed synchronisation/dessynchronisation and convergence/ divergence mechanisms are "one way", in the sense that phase shifts and phase-adjusted correlations can only either increase or diminish across the sample, not both. In view of this, some of the results are not entirely satisfactory. Estimated phase shifts and correlations seem sometimes to be averages of varying patterns within the same regime. In the case of phase-adjusted correlation, the apparent high association of business cycle fluctuations in the 1970's may be masked by the middle of the sample dynamics in some cases. However, the "direction" of both phase effect and phase-adjusted correlation seems in general to be reasonable. The model may not be able to capture the non-monotone time-varying dynamics, it provides an estimate of the predominant path (either increasing or decreasing) of both phase shift and correlation. 

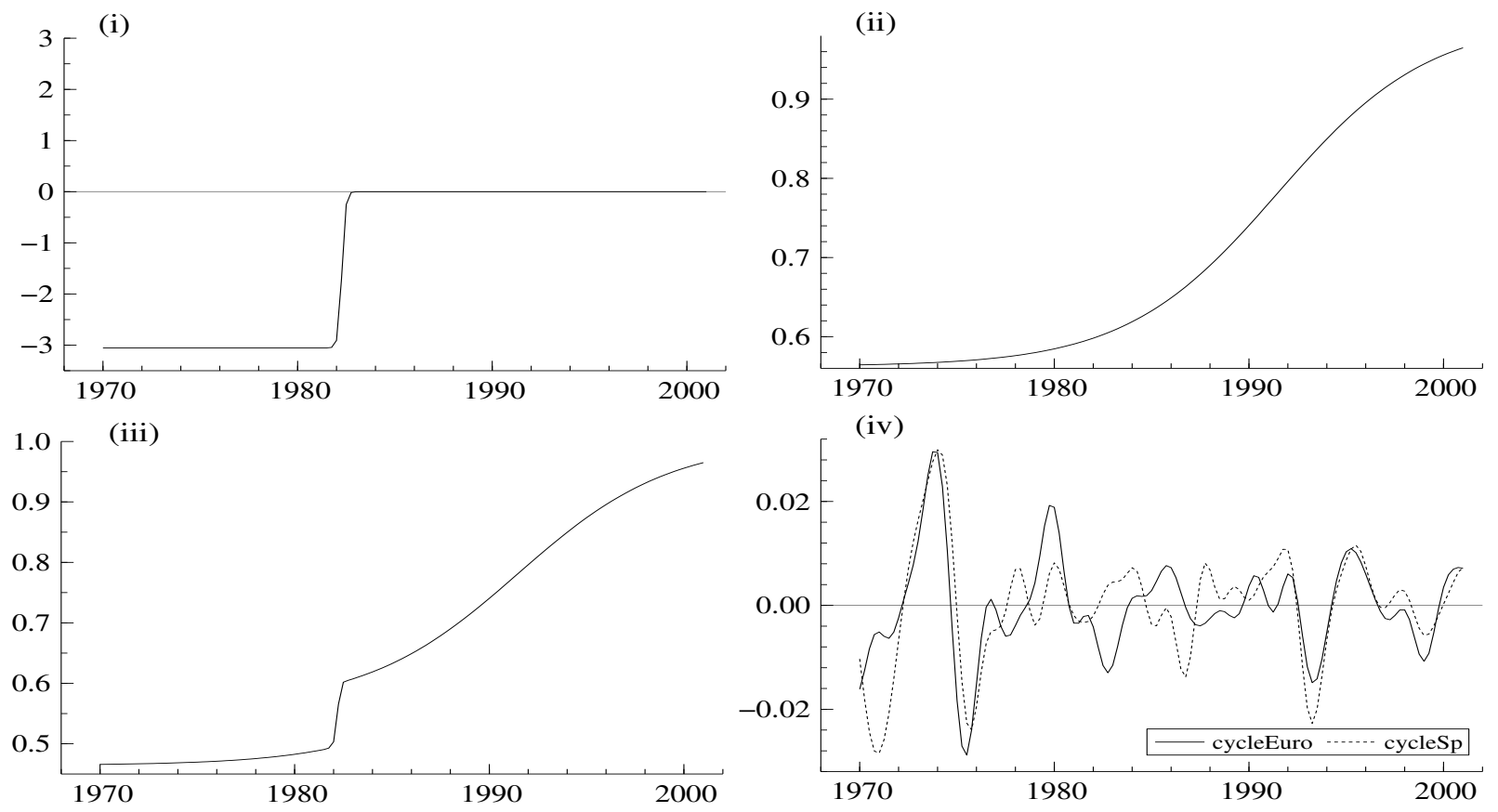

Figure 5: Estimated cycle model for Spain and Euro area: (i) estimated time-varying phase shift; (ii) time-varying phase adjusted correlation; (iii) time-varying contemporaneous correlation; (iv) band-pass filtered series (rescaled)

\section{Conclusions}

Analysis of business cycle comovements among economies is often static. Furthermore, no modelling of relevant features takes place. More or less ad-hoc sub-samples are analysed, parametric and non-parametric statistics computed, and conclusions are drawn. Our modelling strategy avoids many of these shortcomings. Important features and relevant regime switches are estimated. Time-varying phase shifts and time-varying degree of association are explicitly modelled. Transition to different regimes can take place smoothly.

Most results presented are consistent with those of previous studies. Namely, France and Germany display a high degree of association with the Euro area across the sample. Also, their cycles are now synchronised with those of the Euro area, although Germany displayed a leading cycle in the 1970's. Spain, Italy and the Netherlands had a relevant increase in the association with the Euro area, reaching levels of association close to those of Germany and France in the end of the sample. Spain and Italy became more synchronised with the Euro area while The Netherlands displayed a small lead in the end of the sample.

The U.S. leading cycle with respect to the Euro area and the lower degree of association are well established in the literature and were identified by our model. As for the U.K., the estimated synchronisation with the Euro area ocurring in the last years of the sample is worth 

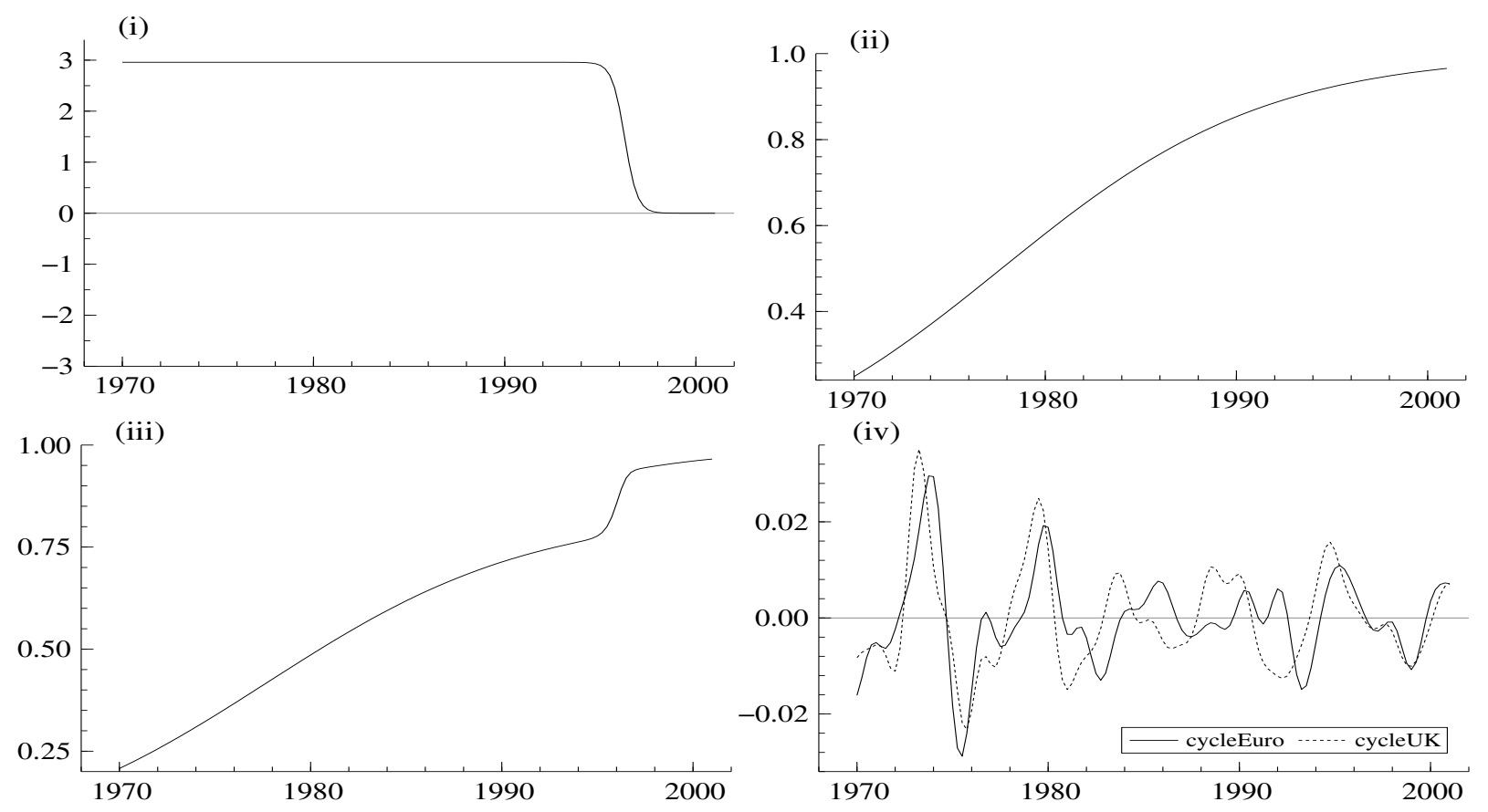

Figure 6: Estimated cycle model for UK and Euro area: (i) estimated time-varying phase shift;

(ii) time-varying phase adjusted correlation; (iii) time-varying contemporaneous correlation; (iv) band-pass filtered series (rescaled)

noting. However, the low degree of association of these two countries with the Euro area reported for the 1970's revealed one of the limitations of the model. Association and phase shifts can only either increase or decrease across a given sample, not both. Future research is planned to increase flexibility in this respect using either deterministic or stochastic functions.

\section{Appendix: stochastic cycles}

In this Appendix we develop the stochastic cycle model for univariate and bivariate time series. In the latter case we discuss the consequences for synchronisation and convergence of two stochastic cycles.

\section{A. Deterministic cycles}

The deterministic cycle $\psi_{t}=a \cos (\lambda t-b)$ with amplitude $a$, phase $b$ and frequency $\lambda$ can be expressed as a sine-cosine wave, that is

$$
\psi_{t}=\alpha \cos (\lambda t)+\beta \sin (\lambda t), \quad t=1, \ldots, n,
$$



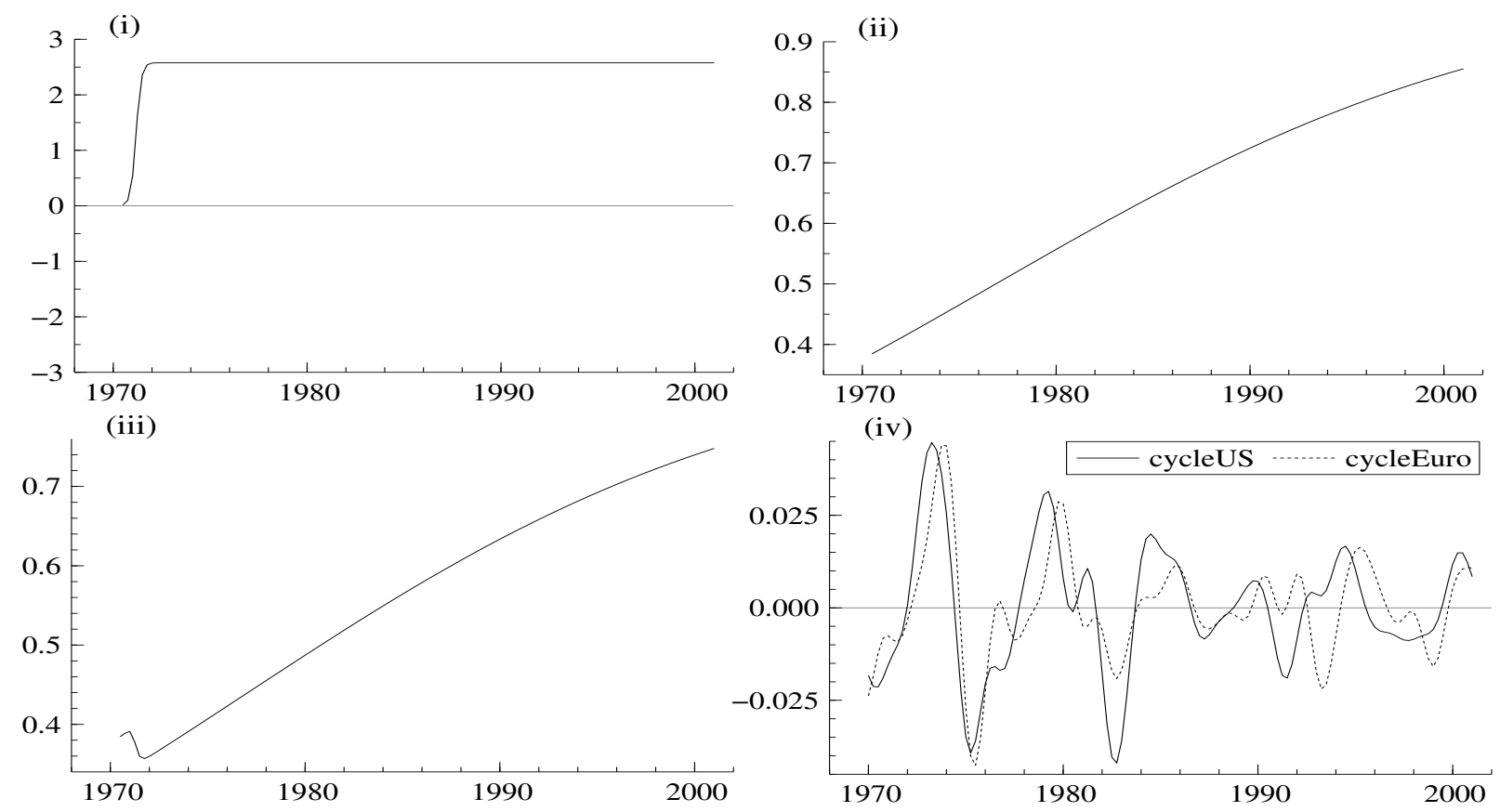

Figure 7: Estimated cycle model for US and Euro area: (i) estimated time-varying phase shift; (ii) time-varying phase adjusted correlation; (iii) time-varying contemporaneous correlation; (iv) band-pass filtered series (rescaled)

where $\alpha=a \cos b$ and $\beta=a \sin b$. The reverse transformation is $a=\alpha^{2}+\beta^{2}$ and $b=\tan ^{-1}(\beta / \alpha)$. The equivalence follows directly from the first of two trigonometric identies

$$
\cos (\lambda \pm \xi)=\cos \lambda \cos \xi \pm \sin \lambda \sin \xi, \quad \sin (\lambda \pm \xi)=\cos \lambda \sin \xi \mp \sin \lambda \cos \xi
$$

with $\xi=b$. The frequency $0 \leq \lambda \leq \pi$ is measured in radians and the period of the cycle is given by $f=2 \pi / \lambda$. The shift is measured in radians as well and the phase shift in time units is given by $b / \lambda$. The cycle can be expressed via a recursion which follows from repeatingly applying the trigonometric identities (15). The recursive expression of the cycle is

$$
\left(\begin{array}{c}
\psi_{t+1} \\
\psi_{t+1}^{+}
\end{array}\right)=\left[\begin{array}{cc}
\cos \lambda & \sin \lambda \\
-\sin \lambda & \cos \lambda
\end{array}\right]\left(\begin{array}{c}
\psi_{t} \\
\psi_{t}^{+}
\end{array}\right),
$$

for $t=0, \ldots, n$ and with $\psi_{0}=\alpha$ and $\psi_{0}^{+}=\beta$. The variable $\psi_{t}^{+}$appears by construction.

The phase shift $b$ is with respect to cycle wave $\cos \lambda t$. Shifts can also be observed between different cycles. Consider two cycles with the same frequency $\lambda$, that is

$$
\psi_{y, t}=\alpha_{y} \cos (\lambda t)+\beta_{y} \sin (\lambda t), \quad \psi_{x, t}=\alpha_{x} \cos (\lambda t)+\beta_{x} \sin (\lambda t) .
$$

In the notation of $(16)$ we have $\psi_{y, t}=(1,0) \bar{\psi}_{y, t}$ and $\psi_{x, t}=(1,0) \bar{\psi}_{x, t}$ where

$$
\bar{\psi}_{y, t+1}=T_{\lambda} \bar{\psi}_{y, t}, \quad \bar{\psi}_{x, t+1}=T_{\lambda} \bar{\psi}_{x, t}
$$


and we define

$$
\bar{\psi}_{y, t}=\left(\begin{array}{c}
\psi_{y, t} \\
\psi_{y, t}^{+}
\end{array}\right), \quad \bar{\psi}_{x, t}=\left(\begin{array}{c}
\psi_{x, t} \\
\psi_{x, t}^{+}
\end{array}\right), \quad T_{\lambda}=\left[\begin{array}{cc}
\cos \lambda & \sin \lambda \\
-\sin \lambda & \cos \lambda
\end{array}\right] .
$$

In the case that phases at the origin of both cycles are equal, that is $\psi_{y, 0}^{+} / \psi_{y, 0}=\psi_{x, 0}^{+} / \psi_{x, 0}$, a phase shift of $\psi_{x, t}$ with respect to $\psi_{y, t}$ can alternatively be established by

$$
\psi_{x, t}=\alpha_{x} \cos [(t-\xi) \lambda]+\beta_{x} \sin [(t-\xi) \lambda],
$$

where $-\frac{1}{2} \pi \leq \xi \lambda \leq \frac{1}{2} \pi$ is measured in radians. The cycle $\psi_{x, t}$ has moved $\xi$ time units "to the right" from cycle $\psi_{y, t}$. In the notation of (17), the shift is obtained by replacing $T_{\lambda}$ by $T_{\lambda-\xi \lambda}$ in (17). More elegantly, the shift can also be accomplished by $\cos (\lambda \xi) \psi_{x, t}+\sin (\lambda \xi) \psi_{x, t}^{+}$to obtain

$$
\psi_{x, t}=\{\cos (\lambda \xi), \sin (\lambda \xi)\} \bar{\psi}_{x, t}, \quad \bar{\psi}_{x, t+1}=T_{\lambda} \bar{\psi}_{x, t},
$$

which follows from the trigonometric identities (15).

\section{B. Stochastic cycles: univariate case}

Following Harvey (1989), a stochastic cycle can be based on (16) with the addition of a damping term $\phi$ and white noise disturbances, that is

$$
\left(\begin{array}{c}
\psi_{t+1} \\
\psi_{t+1}^{+}
\end{array}\right)=\phi\left[\begin{array}{cc}
\cos \lambda & \sin \lambda \\
-\sin \lambda & \cos \lambda
\end{array}\right]\left(\begin{array}{c}
\psi_{t} \\
\psi_{t}^{+}
\end{array}\right)+\left(\begin{array}{c}
\kappa_{t} \\
\kappa_{t}^{+}
\end{array}\right),
$$

where $\kappa_{t}$ and $\kappa_{t}^{+}$are white noise disturbances and mutually independent for $t=1, \ldots, n$. The damping term $0 \leq \phi \leq 1$ ensures that the stochastic process $\psi_{t}$ is stationary. By adopting the notation used in (18) we introduce the Gaussian stationary cycle time series component associated with variable $y$ by

$$
\psi_{y, t}=(1,0) \bar{\psi}_{y, t}, \quad \bar{\psi}_{y, t+1}=\phi T_{\lambda} \bar{\psi}_{y, t}+\bar{\kappa}_{y, t}, \quad \bar{\kappa}_{y, t} \sim \mathcal{N} \mathcal{I} \mathcal{D}\left(0, \sigma_{\kappa, y}^{2} I_{2}\right),
$$

with $\bar{\kappa}_{t}=\left(\kappa_{t}, \kappa_{t}^{+}\right)^{\prime}$ where the two elements of $\bar{\kappa}_{t}$ are independent with the same variances. Time series observations for the variable $y$ may be measured with noise and with a mean different from zero so that

$$
y_{t}=\mu_{y}+\psi_{y, t}+\varepsilon_{y, t}, \quad \varepsilon_{y, t} \sim \mathcal{N} \mathcal{I D}\left(0, \sigma_{\varepsilon, y}^{2}\right), \quad t=1, \ldots, n,
$$

where $y_{t}$ is the observation at time $t$ for variable $y$ with fixed and unknown mean $\mu_{y}$ and observation noise $\varepsilon_{y, t}$ that is mutually independent of $\kappa_{y, t}$ and $\kappa_{y, t}^{+}$at all time points. Stochastic properties of the stationary time series $y_{t}$ are explored in Harvey (1989). The unknown fixed parameters including mean $\mu_{y}$, variances $\sigma_{\varepsilon, y}$ and $\sigma_{\kappa, y}^{2}$, frequency $\lambda$ and damping factor $\phi$ can be based on log-likelihood maximisation using state space methods; see Durbin and Koopman (2001) for a recent discussion. 


\section{Similar stochastic cycles: bivariate case}

The same stochastic cycle model (22) may be adopted for another variable, say $x$. We then have

$$
x_{t}=\mu_{x}+\psi_{x, t}+\varepsilon_{x, t}, \quad \varepsilon_{x, t} \sim \mathcal{N} \mathcal{I} \mathcal{D}\left(0, \sigma_{\varepsilon, x}^{2}\right), \quad t=1, \ldots, n,
$$

with the same definitions for mean $\mu_{x}$, stochastic cycle $\psi_{x, t}$ as in (21) and observation noise $\varepsilon_{x, t}$ as for variable $y$. The cycle disturbances associated with variables $y$ and $x$ are assumed contemporaneously correlated, that is

$$
E\left(\kappa_{y, t} \kappa_{x, t}\right)=E\left(\kappa_{y, t}^{+} \kappa_{x, t}^{+}\right)=\rho_{\kappa} \sigma_{\kappa, y} \sigma_{\kappa, x}, \quad t=1, \ldots, n .
$$

All serial cross-correlations are zero. The cycle of $x$ is said to be similar to $y$ when frequency $\lambda$ and damping factor $\phi$ are restricted to the same values. Stochastic properties of the similar cycle component including variance, autocovariance and cross-autocovariance functions are explored by Harvey and Koopman (1997). For example, the cross-autocovariance function at lag $\tau$ is given by

$$
\gamma_{y, x}(\tau)=\left(1-\phi^{2}\right)^{-1} \phi^{\tau} \cos (\tau \lambda) \rho_{\kappa} \sigma_{\kappa, y} \sigma_{\kappa, x}
$$

for $\tau=1,2, \ldots$.

The stochastic cycle component of variable $x$ can be shifted by modifying the cycle specification for $x_{t}$ as

$$
x_{t}=\mu_{x}+\cos (\lambda \xi) \psi_{x, t}+\sin (\lambda \xi) \psi_{x, t}^{+}+\varepsilon_{x, t}, \quad t=1, \ldots, n,
$$

where $-\frac{1}{2} \pi \leq(\lambda \xi) \leq \frac{1}{2} \pi$ is measured in radians. The same arguments apply as for the shifted deterministic cycle (19). The specification for the cycle of variable $y$ remains the same with common frequency $\lambda$ and damping factor $\phi$. The properties of the similar stochastic cycle component with shifts are explored by Rünstler (2002). For example, the autocovariances $\gamma_{y}(\tau)$ and $\gamma_{x}(\tau)$ and the cross-autocovariance $\gamma_{y, x}(\tau)$ functions at lag $\tau$ are given by

$$
\begin{aligned}
\gamma_{i}(\tau) & =\left(1-\phi^{2}\right)^{-1} \phi^{\tau} \cos (\lambda \tau) \sigma_{\kappa, i}^{2}, \quad i=y, x, \\
\gamma_{y, x}(\tau) & =\left(1-\phi^{2}\right)^{-1} \phi^{\tau} \cos \{\lambda(\tau+\xi)\} \rho_{\kappa} \sigma_{\kappa, y} \sigma_{\kappa, x},
\end{aligned}
$$

for $\tau=1,2, \ldots$. It follows that when $\rho_{\kappa}=0$, cycle cross-autocovariances are zero and do not depend on $\xi$ so that $\xi$ can not be identified in the likelihood function.

In the limiting case of $\mathrm{E}\left(\kappa_{y, t} \kappa_{x, t}\right)=\mathrm{E}\left(\kappa_{y, t}^{+} \kappa_{x, t}^{+}\right)=\sigma_{\kappa, y} \sigma_{\kappa, x}$ and $\rho_{\kappa}=1$, the cycles are said to be common. In this case, the cycle model for $x_{t}$ with shifts can be specified as

$$
x_{t}=\mu_{x}+\delta\left[\cos ((\lambda \xi)) \psi_{y, t}+\sin ((\lambda \xi)) \psi_{y, t}^{+}\right]+\varepsilon_{x, t}, \quad t=1, \ldots, n,
$$

where $\delta$ adjusts for the different amplitude of the cycle of $x_{t}$ with respect to that of $y_{t}$. Although the shifted cycles have common dynamic properties such as the ones described in Engle and Kozicki (1993), the cycles associated with $y_{t}$ and $x_{t}$ have not such properties; see the discussion in Rünstler (2002). 


\section{References}

Angeloni, I. and L. Dedola (1999). From the ERM to the Euro: New evidence on Economic and Policy convergence among EU countries. Working paper, European Central Bank (ECB), Frankfurt.

Artis, M., H. Krolzig, and J. Toro (2002). The European Business cycle. Working paper no. 2242, CEPR, London.

Artis, M. and W. Zhang (1997). International business cycles and the ERM: Is there a European business cycle? International Journal of Finance and Economics 2, 1-16.

Azevedo, J. (2002). Cycle comovement within the European Union in the period 1960-1999. A frequency domain approach. Working paper no. 5-2002, Banco de Portugal.

Azevedo, J. (2003). A generalised Hodrick-Prescott filter: alternative Band-Pass filters for economic time series. Working paper, Free University Amsterdam.

Baxter, M. and R. King (1999). Measuring business cycles: approximate band-pass filters for economic time series. Rev. Economics and Statistics 81, 575-93.

Bayoumi, T. and B. Eichengreen (1993). Shocking aspects of European monetary unification.

In F. Torres and F. Giavazzi (Eds.), The transition to economic and monetary union in Europe, pp. 1-1. Cambridge: Cambridge University Press.

Belo, F. (2001). Some facts about the cyclical convergence in the Euro zone. Working paper no. 7-2001, Banco de Portugal.

Burns, A. and W. Mitchell (1946). Measuring Business cycles. Working paper, NBER, New York.

Chan, W. Y. T. and K. F. Wallis (1978). Multiple time series modelling: another look at the mink-muskrat interactions. Applied Statistics 27, 168-75.

Christiano, L. and T. Fitzgerald (2003). The Band-Pass filter. International Economic Review 44 , forthcoming.

Diebold, F. and G. Rudebusch (1996). Measuring Business cycles: A modern perspective. Rev. Economics and Statistics 78, 67-77.

Doornik, J. A. (2001). Object-Oriented Matrix Programming using Ox 3.0 (4th ed.). London: Timberlake Consultants Ltd. See http://www.nuff.ox.ac.uk/Users/Doornik.

Durbin, J. and S. J. Koopman (2001). Time Series Analysis by State Space Methods. Oxford: Oxford University Press.

ECB (2001). The information content of composite indicators of the Euro area business cycle. Monthly bulletin, November, 39-50, European Central Bank (ECB), Frankfurt. 
Engle, R. F. (2002). Dynamic Conditional Correlation: A simple class of multivariate generalized autoregressive conditional heteroskedasticity models. J. Business and Economic Statist. 20, 339-350.

Engle, R. F. and S. Kozicki (1993). Testing for common features (with discussion). J. Business and Economic Statist. 11, 369-95.

Fagan, G., J. Henry, and R. Mestre (2001). An area wide model for the Euro area. Working paper no. 42, European Central Bank (ECB), Frankfurt.

Hamilton, J. (1989). A new approach to the economic analysis of nonstationary time series and the business cycle. Econometrica 5\%, 357-84.

Harding, D. and A. Pagan (1999). Dissecting the cycles. Working paper, Melbourne Institute, University of Melbourne.

Harvey, A. and T. Trimbur (2003). Generalised model-based filters for extracting trends and cycles in economic time series. Rev. Economics and Statistics 85, 244-255.

Harvey, A. C. (1989). Forecasting, Structural Time Series Models and the Kalman Filter. Cambridge: Cambridge University Press.

Harvey, A. C. and S. J. Koopman (1997). Multivariate structural time series models. In C. Heij, H. Schumacher, B. Hanzon, and C. Praagman (Eds.), Systematic dynamics in economic and financial models, pp. 269-98. Chichester: John Wiley and Sons.

Koopman, S. J., N. Shephard, and J. A. Doornik (1999). Statistical algorithms for models in state space form using SsfPack 2.2. Econometrics Journal 2, 113-66. http://www.ssfpack.com/.

Krolzig, H. and J. Toro (2002). Classical and Modern Business cycle measurement: The European case. Working paper no. 2002/20, Fundacion Centro de Estudios Andaluces, Seville.

Lucas, R. J. (1977). Understanding Business cycles. Stabilization of the domestic and international economy, Carnegie-Rochester Conference Series on Public Policy 5:7-29.

Luginbuhl, R. and S. J. Koopman (2003). Convergence in European GDP series: a multivariate common converging trend-cycle decomposition. Discussion paper no. 03-031, Tinbergen Institute, Amsterdam.

Poirier, D. J. (1976). The Econometrics of Structural Change: with Special Emphasis on Spline Functions. Amsterdam: North-Holland.

Rünstler, G. (2002). Modelling phase shifts among stochastic cycles. Working paper, European Central Bank (ECB), Frankfurt. 
Stock, J. and M. W. Watson (1998). Business cycle fluctuations in U.S. macroeconomic time series. Working paper no. 6528, NBER, New York.

van Dijk, D., T. Terasvirta, and P. H. Franses (2002). Smooth Transition Autoregressive models - A survey of recent developments. Econometric Reviews 21, 1-47.

Wynne, M. and J. Koo (2000). Business cycles under Monetary Union: EU and US business cycles compared. Economica 67, 347-74. 